\title{
Wavelet estimation for derivative of a density in the presence of additive noise
}

\author{
B. L. S. Prakasa Rao \\ CR Rao Advanced Institute of Mathematics, Statistics and Computer Science
}

\begin{abstract}
We construct a wavelet estimator for the derivative of a probability density function in the presence of an additive noise and study its $L_{p}$ consistency property.
\end{abstract}

\section{Introduction}

Methods of nonparametric estimation of a density function and regression function are widely discussed in the literature (cf. Prakasa Rao (1983, 1999a)). It is known that the estimation of derivatives of a density are also of importance and interest to detect possible bumps and to detect monotonicity, concavity or convexity properties of the density function. Asymptotic properties of the kernel type estimators for the derivatives of density have been investigated earlier (cf. Prakasa Rao (1983)).

Our aim in this paper is to discuss wavelet linear estimators for the derivative of a probability density function in the presence of an additive noise. Estimators of density using wavelets was studied for independent and identically distributed random variables in Antoniadis et al. (1994), for some stationary dependent random variables in Leblanc (1996) and for stationary associated sequences in Prakasa Rao (2003). Chaubey et al. $(2006,2008)$ extended these results to derivatives of density estimators for associated sequences and for negatively associated processes. The advantages and disadvantages of the use of wavelet based probability density estimators are discussed in Walter and Ghorai (1992) in the case of independent and identically distributed observations. However, it was shown in Prakasa Rao $(1996,1999 b)$ that one can obtain precise limits on the asymptotic mean integrated squared error for a wavelet based linear estimator for the density function and its derivatives as well as some other functionals of the density (cf. Theorem 3.1, Prakasa Rao (1996)). By "precise limit", we mean that the mean integrated squared error, after suitable scaling, converges to a finite limit as the sample size tends to infinity and this limit can be computed explicitly. Tribouley (1995) studied estimation of multivariate densities using wavelet methods. Prakasa Rao (2000) investigated nonparametric estimation of the partial derivatives of a multivariate probability density. Donoho et al. (1996) investigated density estimation

Key words and phrases. Additive noise, derivative of a probability density function, estimation, mean integrated squared error, nonparametric inference, wavelets.

Received July 2016; accepted July 2017. 
by wavelet thresholding. For a discussion on statistical modeling by wavelets, see Vidakovic (1999).

In recent papers, Chesneau and Doosti (2012) studied wavelet estimation of density for a GARCH model under various dependence structures and Chesneau (2013) investigated wavelet estimation of a density in a GARCH-type model leading to upper bounds on the mean integrated squared error. Shirazi et al. (2012) obtained wavelet based estimation of the derivative of a density by blockthresholding under random censorship. We studied estimation of the derivative of a density in GARCH-type model, which can be considered as a generalization of multiplicative censoring model, in Prakasa Rao (2017). Vardi (1989) (cf. Vardi and Zhang (1992)) introduced the multiplicative censoring model which unifies several models including nonparametric inference for renewal processes, non-parametric deconvolution problems and estimation of decreasing density functions. Chaubey et al. (2015) studied adaptive wavelet estimation of a density from mixtures under multiplicative censoring model generalizing the results in Prakasa Rao (2010). Asgharian et al. (2012) investigated asymptotic properties of the kernel density estimators under multiplicative censoring model. Andersen and Hansen (2001) studied density estimation for multiplicative censoring model using a series expansion approach. Chaubey et al. (2011) give a survey of recent results on linear wavelet density estimation.

Estimation of a probability density function, in the presence of an additive noise, via wavelets has been recently investgated in Li and Liu (2014), Geng and Wang (2015) and Hosseinioun (2016). Density estimation for a statistical model with additive noise plays an important role in statistics and econometrics (cf. Li and Racine (2007)). For earlier work on this problem, see Fan and Koo (2002) and Lounici and Nickl (2011). In practical situations, it is not possible to observe data directly. Suppose we have observed data consisting of independent and identically distributed observations $Y_{1}, \ldots, Y_{n}$ based on the model

$$
Y=X+\varepsilon,
$$

where $X$ is a real valued random variable with unknown probability density function $f_{X}$ and $\varepsilon$ is an independent random noise with a known probabilty density function $g$. The problem of estimation of the density $f_{X}$ based on the observed data $Y_{1}, \ldots, Y_{n}$ has been investigated by the authors cited earlier among others. Our aim is to investigate the problem of estimation of the derivatives of the density $f_{X}$, whenever they exist, based on the observed data $Y_{1}, \ldots, Y_{n}$. As we mentioned earlier, this problem is also of importance and interest to detect possible bumps of the unknown density function $f_{X}$ and to detect monotonicity, concavity or convexity properties of the density function $f_{X}$. Let $f_{Y}$ denote the probability density function of the random variable $Y$. Note that $f_{Y}$ is the convolution of the probability density functions $f_{X}$ and $g$, i.e., $f_{Y}=f_{X} * g$ in the standard notation for convolution. 


\section{Preliminaries on wavelets}

A wavelet system is an infinite collection of translated and scaled versions of functions $\phi(\cdot)$ and $\psi(\cdot)$ called the scaling function and the primary wavelet function respectively. In the following discussion, we assume that $\phi(\cdot)$ is real-valued. The function $\phi(x)$ is a solution of the equation

$$
\phi(x)=\sum_{k=-\infty}^{\infty} C_{k} \phi(2 x-k)
$$

with

$$
\int_{-\infty}^{\infty} \phi(x) d x=1
$$

and the function $\psi(x)$ is defined by

$$
\psi(x)=\sum_{k=-\infty}^{\infty}(-1)^{k} C_{-k+1} \phi(2 x-k) .
$$

The choice of the sequence $\left\{C_{k}\right\}$ determines the wavelet system. It is easy to see that

$$
\sum_{k=-\infty}^{\infty} C_{k}=2
$$

Define

$$
\phi_{j k}(x)=2^{j / 2} \phi\left(2^{j} x-k\right), \quad-\infty<j, k<\infty
$$

and

$$
\psi_{j k}(x)=2^{j / 2} \psi\left(2^{j} x-k\right), \quad-\infty<j, k<\infty .
$$

Suppose the coefficients $\left\{C_{k}\right\}$ satisfy the condition

$$
\begin{aligned}
\sum_{k=-\infty}^{\infty} C_{k} C_{k+2 \ell} & =2 & & \text { if } \ell=0 \\
& =0 & & \text { if } \ell \neq 0 .
\end{aligned}
$$

It is known that, under some additional conditions on $\phi(\cdot)$, the collection $\left\{\psi_{j, k},-\infty<j, k<\infty\right\}$ is an orthonormal basis for $L^{2}(R)$, and $\left\{\phi_{j, k},-\infty<k<\right.$ $\infty$ \} is an orthonormal system in $L^{2}(R)$, for each $-\infty<j<\infty$ (cf. Daubechies (1988, 1992)).

Definition 2.1. The scaling function $\phi$ is said to be $r$-regular for an integer $r \geq 1$, if for every nonnegative integer $\ell \leq r$, and for any integer $k \geq 1$,

$$
\left|\phi^{(\ell)}(x)\right| \leq c_{k}(1+|x|)^{-k}, \quad-\infty<x<\infty
$$

for some $c_{k} \geq 0$ depending only on $k$. Here $\phi^{(\ell)}(\cdot)$ denotes the $\ell$ th derivative of $\phi(\cdot)$. 
Definition 2.2. A multiresolution analysis of $L^{2}(R)$ consists of an increasing sequence of closed subspaces $\left\{V_{j}\right\}$ of $L^{2}(R)$ such that

(i) $\bigcap_{j=-\infty}^{\infty} V_{j}=\{0\}$;

(ii) $\bigcup_{j=-\infty}^{\infty} V_{j}=L^{2}(R)$;

(iii) there is a scaling function $\phi \in V_{0}$ such that $\{\phi(x-k),-\infty<k<\infty\}$ is an orthonormal basis for $V_{0}$;

(iv) for all $h(\cdot) \in L^{2}(R),-\infty<k<\infty, h(x) \in V_{0} \Rightarrow h(x-k) \in V_{0}$; and

(v) $h(\cdot) \in V_{j} \Rightarrow h(2 x) \in V_{j+1}$.

Mallat (1989) has shown that, given any multiresolution analysis, it is possible to find a function $\psi(\cdot)$ (called primary wavelet function) such that, for any fixed $j,-\infty<j<\infty$, the family $\left\{\psi_{j, k},-\infty<k<\infty\right\}$ is an orthonormal basis of the orthogonal complement $W_{j}$ of $V_{j}$ in $V_{j+1}$ so that $\left\{\psi_{j, k},-\infty<j, k<\infty\right\}$ is an orthonormal basis of $L^{2}(R)$ (cf. Daubechies $(1988,1992)$ ). When the scaling function $\phi(\cdot)$ is $r$-regular, the corresponding multiresolution analysis is said to be $r$-regular.

Let $f \in L_{2}(R)$. The function $f$ can be expanded in the form (cf. Daubechies (1992)):

$$
f=\sum_{k=-\infty}^{\infty} a_{s, k} \phi_{s, k}+\sum_{j=s}^{\infty} \sum_{k=-\infty}^{\infty} b_{j, k} \psi_{j, k}
$$

for any integer $-\infty<s<\infty$. Observe that the wavelet coefficients are given by

$$
a_{s, k}=\int_{-\infty}^{\infty} f(x) \phi_{s, k}(x) d x
$$

and

$$
b_{j, k}=\int_{-\infty}^{\infty} f(x) \psi_{j, k}(x) d x
$$

Suppose that the functions $\phi$ and $\psi$ belong to $C^{r}$, the space of functions with $r$ continuous derivatives for some $r \geq 1$, and have compact support contained in an interval $[-\delta, \delta]$ for some $\delta>0$. It follows, from the Corollary 5.5.2 in Daubechies (1992), that the function $\psi(\cdot)$ is orthogonal to polynomials of degree less than or equal to $r$. In particular

$$
\int_{-\infty}^{\infty} \psi(x) x^{\ell} d x=0, \quad \ell=0,1, \ldots, r .
$$

This brief discussion on wavelets is based on Antoniadis et al. (1994). For more details, see Daubechies (1992) and Strang (1989). 


\section{More on wavelets}

Let $\phi(\cdot)$ be a scaling function as defined earlier. Define

$$
\theta_{\phi}(x)=\sum_{k=-\infty}^{\infty}|\phi(x-k)| \text {. }
$$

Suppose the following conditions hold:

$(\mathcal{C} 1)$ The $\operatorname{ess} \sup _{x} \theta_{\phi}(x)<\infty$ where

$$
\text { ess } \sup _{x} g(x)=\inf \{y: \lambda([x: g(x)>y])=0\}
$$

and $\lambda$ is the Lebesgue measure on the real line.

$(\mathcal{C} 2)$ There exists a bounded nonincreasing function $\Phi(\cdot)$ such that $|\phi(u)| \leq$ $\Phi(|u|)$ almost every where and

$$
\int_{0}^{\infty}|u|^{r} \Phi(|u|) d u<\infty
$$

for some integer $r \geq 0$.

The following Lemmas 3.1 to 3.3 follow from the results in Hardle et al. (1998).

Lemma 3.1. Suppose the condition (C1) holds. Then, for any sequence $\left\{\lambda_{s}\right.$, $s \in \mathcal{Z}\} \in \ell_{p}$,

$$
C_{1}\|\lambda\|_{\ell_{p}} 2^{\frac{s}{2}-\frac{s}{p}} \leq\left\|\sum_{k} \lambda_{k} \phi_{s, k}\right\|_{p} \leq C_{2}\|\lambda\|_{\ell_{p}} 2^{\frac{s}{2}-\frac{s}{p}},
$$

where

$$
C_{1}=\left(\left\|\theta_{\phi}\right\|_{\infty}^{1 / p}\|\phi\|_{1}^{1 / q}\right)^{-1}
$$

and

$$
C_{2}=\left(\left\|\theta_{\phi}\right\|_{\infty}^{1 / q}\|\phi\|_{1}^{1 / p}\right)^{-1},
$$

where $1 \leq p \leq \infty, \frac{1}{p}+\frac{1}{q}=1$ with suitable interpretation for $p$ and $q$ in the boundary case.

Since the scaling function $\phi$ satisfies the condition $(\mathcal{C} 1)$, the kernel function

$$
K(x, y)=\sum_{k} \phi(x-k) \phi(y-k)
$$

is well defined and it is called the orthonormal projection associated with the function $\phi$. Let

$$
K_{s}(x, y)=2^{s} K\left(2^{s} x, 2^{s} y\right)
$$


for any integer $s \geq 0$. For any function $h \in L_{p}(R), 1 \leq p \leq \infty$, define

$$
K_{s} h(x)=\int_{-\infty}^{\infty} K_{s}(x, y) h(y) d y=\sum_{s} \alpha_{s, k} \phi_{s, k}(x),
$$

where

$$
\alpha_{s, k}=\int_{-\infty}^{\infty} \phi_{s, k}(x) h(x) d x
$$

Lemma 3.2. Suppose the condition (C2) holds. Then

$$
\int_{-\infty}^{\infty} K(x, y) d y=1 \quad \text { a.e. }
$$

and

$$
\text { (ii) } \quad|K(x, y)| \leq C_{1} \Phi\left(\frac{|x-y|}{C_{2}}\right) \quad \text { a.e., }
$$

where $C_{1}$ and $C_{2}$ are positive constants depending on $\Phi$.

Let $F(x)=C_{1} \Phi\left(\frac{|x|}{C_{2}}\right)$. Then the function $F \in L_{1}(R) \cap L_{\infty}(R)$ and $|K(x, y)| \leq$ $F(x-y)$ a.e.

Lemma 3.3. Suppose the condition (C2) holds and $h \in L_{p}(R), 1 \leq p<\infty$. Then

$$
\lim _{n \rightarrow \infty}\left\|K_{s} h-h\right\|_{p}=0
$$

Suppose the function $h^{(d)}$ exists and $h^{(d)} \in L_{p}(R)$ for some $1 \leq p<\infty$. As a consequence of Lemma 3.3, it follows that

$$
\lim _{n \rightarrow \infty}\left\|K_{s} h^{(d)}-h^{(d)}\right\|_{p}=0
$$

It can be shown that Lemma 3.3 holds for $h \in L_{\infty}(R)$ if the function $h(\cdot)$ is uniformly continuous. We will now state another result known as Rosenthal's inequality (Rosenthal (1970)) which will be used in the sequel.

Lemma 3.4. Let $X_{1}, \ldots, X_{n}$ be independent random variables with mean zero and further suppose that $\left|X_{i}\right| \leq M<\infty, 1 \leq i \leq n$. Then there exists a constant $C_{p}>0$, such that

$$
E\left(\left|\sum_{i=1}^{n} X_{i}\right|^{p}\right) \leq C_{p}\left(M^{p-2} \sum_{i=1}^{n} E\left(X_{i}^{2}\right)+\left(\sum_{i=1}^{n} E\left(X_{i}^{2}\right)\right)^{p / 2}\right), \quad p>2,
$$

and

$$
\text { (ii) } \quad E\left(\left|\sum_{i=1}^{n} X_{i}\right|^{p}\right) \leq C_{p}\left(\sum_{i=1}^{n} E\left(X_{i}^{2}\right)\right)^{p / 2}, \quad 0<p \leq 2 \text {. }
$$




\section{Estimation of the $d$ th derivative of a probability density function}

For any function $h(\cdot) \in L_{1}(R)$, define the Fourier transform

$$
\tilde{h}(t)=\int_{-\infty}^{\infty} h(x) e^{-i t x} d x, \quad-\infty<t<\infty .
$$

It is known that $\tilde{f}_{Y}(t)=\tilde{f}_{X}(t) \tilde{g}(t), t \in R$. Suppose that the Fourier transform $\tilde{g}(t)$ of the probability density function $g$ is non-vanishing for all $t \in R$.

Let $\left\{X_{i}, 1 \leq i \leq n\right\}$ be independent and identically distributed random variables with probability density function $f_{X}$ which is $d$-times differentiable. Suppose that the derivative $f_{X}^{(d)}$ of $f_{X}$ exists, bounded, has compact support and $f_{X}^{(d)} \in L_{2}(R)$. Let us first consider the estimation of the probability density function $f_{X}$. A wavelet based density estimator of the density function $f_{X}$ can be motivated in the following way from the expansion given in the equation (2.9) (cf. Prakasa Rao (2003)). We can estimate $f_{X}(x)$ by $\hat{f}_{X}(x)$ where

$$
\hat{f}_{X}(x)=\sum_{k \in N_{s}} \alpha_{s, k} \phi_{s, k}(x),
$$

where

$$
\alpha_{s, k}=\frac{1}{n} \sum_{i=1}^{n} \phi_{s, k}\left(X_{i}\right) .
$$

Here $N_{s}$ is the set of integers $k$ such that $\operatorname{supp}\left(f_{X}\right) \cap \operatorname{supp}\left(\phi_{s, k}\right)$ is nonempty. Since the functions $f_{X}$ and $\phi$ have compact supports, the cardinality of the set $N_{S}$ is finite and it is of the order $O\left(2^{s}\right)$.

Let us now consider the problem of estimation of the derivative $f_{X}^{(d)}$ of $f_{X}$. As in Prakasa Rao (1996), we assume that $f_{X}^{d} \in L_{2}(R)$ and that there exist $D_{j} \geq 0, \beta_{j} \geq 0$, such that

$$
\left|f_{X}^{(j)}(x)\right| \leq D_{j}|x|^{-\beta_{j}}, \quad|x| \geq 1,0 \leq j \leq d,
$$

where $\beta_{0}>4 d+1$. Suppose the multiresolution analysis generated by the scaling function $\phi$ is $r$-regular for some $r \geq d$. Then, by definition, $\phi \in C^{(r)}, \phi$ and its derivatives $\phi^{(j)}$ up to order $r$ are rapidly decreasing, that is, for every integer $m \geq 1$, there exists a constant $A_{m}>0$, such that

$$
\left|\phi^{(j)}(x)\right| \leq A_{m}(1+|x|)^{-m}, \quad 0 \leq j \leq r .
$$

If $d \geq 1$, then it is clear that

$$
\lim _{|x| \rightarrow \infty} \phi_{s, k}^{(j)}(x) f^{(d-j-1)}(x)=0, \quad 0 \leq j \leq d-1
$$

for any fixed $s$ and $k$. The projection of $f_{X}^{(d)}$ on $V_{s}$ is

$$
f_{X, s}^{(d)}(x)=\sum_{k \in N_{s}} a_{s, k} \phi_{s, k}(x),
$$


where

$$
\begin{aligned}
a_{s, k} & =\int_{-\infty}^{\infty} f_{X}^{(d)}(x) \phi_{s, k}(x) d x \\
& =(-1)^{d} \int_{-\infty}^{\infty} f_{X}(x) \phi_{s, k}^{(d)}(x) d x .
\end{aligned}
$$

The last equality given above can be justified by using integration by parts since the function $\phi(\cdot)$ is $r$-regular (cf. Prakasa Rao (1996)). This expression motivates the following estimator for $f_{X}^{(d)}(x)$ :

$$
\tilde{f}_{X, s}^{(d)}(x)=\sum_{k \in N_{s}} a_{s, k}^{*} \phi_{s, k}(x),
$$

where

$$
a_{s, k}^{*}=\frac{(-1)^{d}}{n} \sum_{i=1}^{n} \phi_{s, k}^{(d)}\left(X_{i}\right) .
$$

Note that the estimator defined above in the equation (4.5) reduces to the density estimator given in (4.1) for $d=0$. Since the random sample $X_{i}, 1 \leq i \leq n$ is unobservable and the observed data is $Y_{i}=X_{i}+\varepsilon_{i}, 1 \leq i \leq n$, we now modify the estimator $\tilde{f}_{X, s}^{(d)}(x)$.

By Plancherel formula,

$$
\begin{aligned}
a_{s, k} & =(-1)^{d} \int_{-\infty}^{\infty} f_{X}(x) \phi_{s, k}^{(d)}(x) d x \\
& =\frac{(-1)^{d}}{2 \pi} \int_{-\infty}^{\infty} \tilde{f}_{X}(t) \overline{\tilde{\phi}_{s, k}^{(d)}(t)} d t \\
& =\frac{(-1)^{d}}{2 \pi} \int_{-\infty}^{\infty} \frac{\tilde{f}_{Y}(t)}{\tilde{g}(t)} \tilde{\phi}_{s, k}^{(d)}(-t) d t .
\end{aligned}
$$

For any function $\psi(\cdot)$ which is $d$-times differentiable, define $\psi_{s, k}(x)=2^{s / 2} \times$ $\psi\left(2^{s} x-k\right)$ for integers $s, k$ and let $\psi_{s, k}^{(d)}(x)$ denote the $d$ th derivative of the function $\psi_{s, k}(x)$. Define the operator $H_{s}$ by the transformation

$$
\left(H_{s} \psi^{(d)}\right)_{s, k}(y)=\frac{1}{2 \pi} \int_{R} e^{i t y} \frac{\tilde{\psi}_{s, k}^{(d)}(t)}{\tilde{g}(-t)} d t, \quad y \in R
$$

for all integers $-\infty<s, k<\infty$. It can be checked that

$$
\tilde{\psi}_{s, k}^{(d)}(u)=e^{i k u 2^{s}} 2^{-d s+\frac{s}{2}} \tilde{\psi}_{s, k}^{(d)}\left(u 2^{s}\right)
$$

which we will use in the computations later. Let

$$
\hat{a}_{s, k}=\frac{(-1)^{d}}{n} \sum_{i=1}^{n}\left(H_{s} \phi^{(d)}\right)_{s, k}\left(Y_{i}\right)
$$


We will consider the modified estimator $\hat{f}_{X, s}^{(d)}(x)$ defined by

$$
\begin{aligned}
\hat{f}_{X, s}^{(d)}(x) & =\sum_{k \in N_{s}} \hat{a}_{s, k} \phi_{s, k}(x) \\
& =\sum_{k \in N_{s}}\left[\frac{(-1)^{d}}{n} \sum_{i=1}^{n}\left(H_{s} \phi^{(d)}\right)_{s, k}\left(Y_{i}\right)\right] \phi_{s, k}(x) \\
& =\frac{(-1)^{d}}{n} \sum_{i=1}^{n}\left[\sum_{k \in N_{s}}\left(H_{s} \phi^{(d)}\right)_{s, k}\left(Y_{i}\right) \phi_{s, k}(x)\right]
\end{aligned}
$$

as an estimator of $f_{X}^{(d)}(x)$.

Lemma 4.1. If the function $f_{X}^{(d)} \in L_{2}(R)$, then the estimator $\hat{a}_{s, k}$ defined by the equation (4.6) is an unbiased estimator of the wavelet coefficient $a_{s, k}$ given by the equation (4.4).

Proof. Note that

$$
\begin{aligned}
& E\left[\hat{a}_{s, k}\right]=E\left[\frac{(-1)^{d}}{n} \sum_{i=1}^{n}\left(H_{s} \phi^{(d)}\right)_{s, k}\left(Y_{i}\right)\right] \\
& =(-1)^{d} E\left[\left(H_{s} \phi^{(d)}\right)_{s, k}\left(Y_{1}\right)\right] \\
& =\frac{(-1)^{d}}{2 \pi} \int_{-\infty}^{\infty}\left[\int_{-\infty}^{\infty} e^{i t y} \frac{\tilde{\phi}_{s, k}^{(d)}(t)}{\tilde{g}(-t)} d t\right] f_{Y}(y) d y \\
& =\frac{(-1)^{d}}{2 \pi} \int_{-\infty}^{\infty}\left[\int_{-\infty}^{\infty} e^{i t y} f_{Y}(y) d y\right] \frac{\tilde{\phi}_{s, k}^{(d)}(t)}{\tilde{g}(-t)} d t \\
& =\frac{(-1)^{d}}{2 \pi} \int_{-\infty}^{\infty} \tilde{f}_{Y}(-t) \frac{\tilde{\phi}_{s, k}^{(d)}(t)}{\tilde{g}(-t)} d t \\
& =\frac{(-1)^{d}}{2 \pi} \int_{-\infty}^{\infty} \tilde{f}_{X}(-t) \tilde{\phi}_{s, k}^{(d)}(t) d t \\
& =\frac{(-1)^{d}}{2 \pi} \int_{-\infty}^{\infty} \tilde{f}_{X}(t) \tilde{\phi}_{s, k}^{(d)}(-t) d t \\
& =\frac{(-1)^{d}}{2 \pi} \int_{-\infty}^{\infty} \tilde{f}_{X}(t) \overline{\tilde{\phi}_{s, k}^{(d)}(t)} d t \\
& =(-1)^{d} \int_{-\infty}^{\infty} f_{X}(x) \phi_{s, k}^{(d)}(x) d x \\
& =\int_{-\infty}^{\infty} f_{X}^{(d)}(x) \phi_{s, k}(x) d x \\
& =a_{s, k} \text {. }
\end{aligned}
$$


We will now discuss $L_{p}$-consistency of the estimator $\hat{f}_{X, s}^{(d)}(x)$ for estimating of the function $f_{X}^{(d)}(x)$ following the techniques in Geng and Wang (2015). For any function $f \in L_{p}(R)$, we write $\|f\|_{p}^{p}$ for $\int_{R}|f(x)|^{p} d x$.

Theorem 4.1. Suppose that $\tilde{g}(t) \simeq\left(1+\left|t^{2}\right|\right)^{-\beta / 2}, t \in R$ for some $\beta \geq 0$ and the function $f_{X}^{(d)} \in L_{p}(R)$ for some $2 \leq p<\infty$. Further suppose that $f_{Y} \in L_{p / 2}(R)$. Choose the positive integer s such that $2^{s} \simeq n^{\frac{1-\varepsilon}{1+2 \beta+4 d \frac{2 p-1}{p}}}$ for some $0<\varepsilon<1$. Define the estimator $\hat{f}_{X, s}^{(d)}(x)$ as an estimator of the function $f_{X}^{(d)}(x)$. Then the estimator $\hat{f}_{X, s}^{(d)}(x)$ is $L_{p}$-consistent, that is,

$$
\lim _{n \rightarrow \infty} E\left\|\hat{f}_{X, s}^{(d)}-f_{X}^{(d)}\right\|_{p}=0
$$

Proof. Note that

$$
\begin{aligned}
E\left[\hat{f}_{X, s}^{(d)}(x)\right] & =E\left[\sum_{k \in N_{s}} \hat{a}_{s, k} \phi_{s, k}(x)\right] \\
& =E\left[\sum_{k \in N_{s}}\left[\frac{(-1)^{d}}{n} \sum_{i=1}^{n}\left(H_{s} \phi^{(d)}\right)_{s, k}\left(Y_{i}\right)\right] \phi_{s, k}(x)\right] \\
& =E\left[(-1)^{d} \sum_{k \in N_{s}}\left(H_{s} \phi^{(d)}\right)_{s, k}\left(Y_{1}\right)\right] \phi_{s, k}(x) \\
& =(-1)^{d} \sum_{k \in N_{s}} E\left[\left(H_{s} \phi^{(d)}\right)_{s, k}\left(Y_{1}\right)\right] \phi_{s, k}(x) \\
& =\sum_{k \in N_{s}} a_{s, k} \phi_{s, k}(x) \\
& =K_{s} f_{X}^{(d)}(x)
\end{aligned}
$$

where the operator $K_{S}$ is as defined by the equation (3.1).

As a consequence of the equation (3.2) following Lemma 3.3 (cf. Hardle et al. (1998)), it follows that

$$
\lim _{n \rightarrow \infty}\left\|f_{X}^{(d)}-E\left[\hat{f}_{X, s}^{(d)}\right]\right\|_{p}=\lim _{n \rightarrow \infty}\left\|f_{X}^{(d)}-K_{s} f_{X}^{(d)}\right\|_{p}=0
$$

In the following discussion, we will denote $A_{s} \simeq B_{s}$ if there exist positive constants $C_{1}$ and $C_{2}$ such that

$$
C_{1} B_{s} \leq A_{s} \leq C_{2} B_{s}
$$

as $s \rightarrow \infty$. We will now estimate the term

$$
\left\|\hat{f}_{X, s}^{(d)}-E\left[\hat{f}_{X, s}^{(d)}\right]\right\|_{p}
$$


Note that

$$
\begin{aligned}
\left\|\hat{f}_{X, s}^{(d)}-E\left[\hat{f}_{X, s}^{(d)}\right]\right\|_{p}^{p} & \left.=\| \sum_{k \in N_{s}} \hat{a}_{s, k} \phi_{s, k}(x)-\sum_{k \in N_{s}} a_{s, k} \phi_{s, k}(x)\right] \|_{p}^{p} \\
& =\left\|\sum_{k \in N_{s}}\left(\hat{a}_{s, k}-a_{s, k}\right) \phi_{s, k}(x)\right\|_{p}^{p} \\
& \simeq 2^{s\left(\frac{p}{2}-1\right)}\left[\sum_{k \in N_{s}}\left|\hat{a}_{s, k}-a_{s, k}\right|^{p}\right] \quad \text { (by Lem }
\end{aligned}
$$

and hence

$$
\begin{aligned}
E\left[\left\|\hat{f}_{X, s}^{(d)}-E\left[\hat{f}_{X, s}^{(d)}\right]\right\|_{p}^{p}\right] & \simeq 2^{s\left(\frac{p}{2}-1\right)} E\left[\sum_{k \in N_{s}}\left|\hat{a}_{s, k}-a_{s, k}\right|^{p}\right] \\
& =2^{s\left(\frac{p}{2}-1\right)}\left[\sum_{k \in N_{s}} E\left|\hat{a}_{s, k}-a_{s, k}\right|^{p}\right] .
\end{aligned}
$$

Observe that

$$
\begin{aligned}
\left|\hat{a}_{s, k}-a_{s, k}\right| & =\left|\frac{1}{n} \sum_{i=1}^{n}\left(H_{s} \phi^{(d)}\right)_{s, k}\left(Y_{i}\right)-\frac{1}{n} \sum_{i=1}^{n} E\left[\left(H_{s} \phi^{(d)}\right)_{s, k}\left(Y_{i}\right)\right]\right| \\
& =\frac{1}{n}\left|\sum_{i=1}^{n} Z_{i k}\right|,
\end{aligned}
$$

where

$$
Z_{i k}=\left(H_{s} \phi^{(d)}\right)_{s, k}\left(Y_{i}\right)-E\left[\left(H_{s} \phi^{(d)}\right)_{s, k}\left(Y_{i}\right)\right]
$$

Therefore

$$
\begin{aligned}
\left|\left(H_{s} \phi^{(d)}\right)_{s, k}(y)\right| & =\left|\frac{1}{2 \pi} \int_{R} e^{i t y} \frac{\tilde{\phi}_{s, k}^{(d)}(t)}{\tilde{g}(-t)} d t\right| \\
& \leq \frac{1}{2 \pi} \int_{R}\left|\frac{\tilde{\phi}_{s, k}^{(d)}(t)}{\tilde{g}(-t)}\right| d t \\
& \simeq \frac{1}{2 \pi} \int_{R}\left|\tilde{\phi}_{s, k}^{(d)}(t)\right|(1+|t|)^{\beta / 2} d t \\
& \simeq 2^{d s-\frac{s}{2}} \int_{R}\left|\tilde{\phi}^{(d)}(u)\right|\left(1+|u| 2^{s}\right)^{\beta / 2} 2^{s} d u \\
& \simeq 2^{d s+\frac{s}{2}} 2^{\beta s} .
\end{aligned}
$$


Hence,

$$
\begin{aligned}
\left|Z_{i k}\right|= & \left|\left(H_{s} \phi^{(d)}\right)_{s, k}\left(Y_{i}\right)-E\left[\left(H_{s} \phi^{(d)}\right)_{s, k}\left(Y_{i}\right)\right]\right| \\
\leq & \left|\left(H_{s} \phi^{(d)}\right)_{s, k}\left(Y_{i}\right)\right|+E\left|\left(H_{s} \phi^{(d)}\right)_{s, k}\left(Y_{i}\right)\right| \\
= & \left|\frac{1}{2 \pi} \int_{-\infty}^{\infty} 2^{d s+\frac{s}{2}} e^{i t\left(2^{Y_{i}}-k\right)} \frac{\tilde{\phi}^{(d)}(t)}{\tilde{g}\left(-2^{s} t\right)} d t\right| \\
& +\frac{1}{2 \pi} \int_{-\infty}^{\infty}\left|\int_{-\infty}^{\infty} 2^{d s+\frac{s}{2}} e^{i t\left(2^{y}-k\right)} \frac{\tilde{\phi}^{(d)}(t)}{\tilde{g}\left(-2^{s} t\right)} d t\right| f_{y}(y) d y \\
\simeq & 2^{s\left(\frac{1}{2}+\beta+d\right)} .
\end{aligned}
$$

Applying Rosenthal's inequality (Lemma 3.4), it follows that

$$
\begin{aligned}
E\left[\left|\hat{a}_{s, k}-a_{s, k}\right|^{p}\right] & =\frac{1}{n^{p}} E\left|\sum_{i=1}^{n} Z_{i k}\right|^{p} \\
& \simeq \frac{1}{n^{p}}\left[2^{s\left(\frac{1}{2}+\beta+d\right)(p-2)} \sum_{i=1}^{n} E\left|Z_{i k}\right|^{2}+\left(\sum_{i=1}^{n} E\left|Z_{i k}\right|^{2}\right)^{p / 2}\right] \\
& =\frac{2^{s\left(\frac{1}{2}+\beta+d\right)(p-2)}}{n^{p-1}} E\left|Z_{1 k}\right|^{2}+\frac{1}{n^{p / 2}}\left(E\left|Z_{1 k}\right|^{2}\right)^{p / 2} .
\end{aligned}
$$

We will now estimate $\sum_{k}\left(E\left|Z_{1 k}\right|^{2}\right)^{p / 2}$. Observe that

$$
\begin{aligned}
A & =\int_{R}\left|\left(H_{s} \phi^{(d)}\right)_{s, k}(y)\right|^{2} d y \\
& =2 \pi \int_{R}\left|\frac{\tilde{\phi}_{s, k}^{(d)}(t)}{\tilde{g}(-t)}\right|^{2} d t \\
& \simeq 2^{4 d s-s} \int_{R}\left|\frac{\tilde{\phi}^{(d)}\left(t 2^{-s}\right)}{\tilde{g}(-t)}\right|^{2} d t \\
& \simeq 2^{s(4 d-1)} \int_{R}\left|\frac{\tilde{\phi}^{(d)}(u)}{\tilde{g}\left(-u 2^{s}\right)}\right|^{2} 2^{s} d u \\
& \simeq 2^{4 d s} \int_{R}\left|\left(1+\left|u^{2} 2^{2 s}\right|\right)^{\beta / 2} \tilde{\phi}^{(d)}(u)\right|^{2} d u \\
& \simeq 2^{4 d s+2 \beta s} .
\end{aligned}
$$

Hence,

$$
\begin{aligned}
\left(E\left|Z_{1 k}\right|^{2}\right)^{p / 2} & =\left(E\left|\left(H_{s} \phi^{(d)}\right)_{s, k}\left(Y_{1}\right)-E\left[\left(H_{s} \phi^{(d)}\right)_{s, k}\left(Y_{1}\right)\right]\right|^{2}\right)^{p / 2} \\
& \leq\left(E\left|\left(H_{s} \phi^{(d)}\right)_{s, k}\left(Y_{1}\right)\right|^{2}\right)^{p / 2}
\end{aligned}
$$




$$
\begin{aligned}
& =\left(\int_{R}\left|\left(H_{S} \phi^{(d)}\right)_{s, k}(y)\right|^{2} f_{Y}(y) d y\right)^{p / 2} \\
& =A^{p / 2}\left(\int_{R}\left|\frac{\left(H_{s} \phi^{(d)}\right)_{s, k}(y)}{A}\right|^{2} f_{Y}(y) d y\right)^{p / 2} \\
& \leq A^{\frac{p}{2}-1}\left(\int_{R}\left|\left(H_{S} \phi^{(d)}\right)_{s, k}(y)\right|^{2}\right)\left(f_{Y}(y)\right)^{p / 2} d y .
\end{aligned}
$$

Furthermore,

$$
\begin{aligned}
& \sum_{k}\left|\left(H_{S} \phi^{(d)}\right)_{s, k}(y)\right|^{2}=\sum_{k}\left|\frac{1}{2 \pi} \int_{R} e^{i t y} \frac{\tilde{\phi}_{s, k}^{(d)}(t)}{\tilde{g}(-t)} d t\right|^{2} \\
& \simeq \sum_{k}\left(2^{d s-s / 2}\left|\int_{-4 \pi / 3}^{4 \pi / 3} e^{i t(y-k)} \frac{\tilde{\phi}^{(d)}\left(t 2^{-s}\right)}{\tilde{g}(-t)} d t\right|\right)^{2} \\
& \simeq 2^{2 d s-s} \sum_{k}\left(\left|\int_{-4 \pi / 3}^{4 \pi / 3} e^{i t(y-k)} \frac{\tilde{\phi}^{(d)}\left(t 2^{-s}\right)}{\tilde{g}(-t)} d t\right|\right)^{2} \\
& =2^{2 d s-s} \sum_{k}\left(\left|\int_{-4 \pi / 3}^{4 \pi / 3} e^{i(y-k) u 2^{s}} \frac{\tilde{\phi}^{(d)}(u)}{\tilde{g}\left(-u 2^{s}\right)} 2^{s} d u\right|\right)^{2} \\
& =2^{2 d s+s} \sum_{k}\left(\left|\int_{-4 \pi / 3}^{4 \pi / 3} e^{i(y-k) u 2^{s}} \frac{\tilde{\phi}^{(d)}(u)}{\tilde{g}\left(-u 2^{s}\right)} d u\right|\right)^{2} \\
& =2^{2 d s+s} \sum_{k}\left(\left|\int_{0}^{4 \pi / 3} e^{i t 2^{s} y} \frac{\tilde{\phi}^{(d)}(t)}{\tilde{g}\left(-2^{s} t\right)} e^{-i t 2^{s} k} d t\right|\right. \\
& \left.+\left|\int_{-4 \pi / 3}^{0} e^{i t 2^{s} y} \frac{\tilde{\phi}^{(d)}(t)}{\tilde{g}\left(-2^{s} t\right)} e^{-i t 2^{s} k} d t\right|\right)^{2} \\
& \simeq 2^{2 d s+s}\left[\sum_{k}\left|\int_{0}^{4 \pi / 3} e^{i t 2^{s} y} \frac{\tilde{\phi}^{(d)}(t)}{\tilde{g}\left(-2^{s} t\right)} e^{-i t 2^{s} k} d t\right|^{2}\right. \\
& \left.+\sum_{k}\left|\int_{-4 \pi / 3}^{0} e^{i t 2^{s} y} \frac{\tilde{\phi}^{(d)}(t)}{\tilde{g}\left(-2^{s} t\right)} e^{-i t 2^{s} k} d t\right|^{2}\right]
\end{aligned}
$$

Observe that the function

$$
e^{i t 2^{s} y} \frac{\tilde{\phi}^{(d)}(t)}{\tilde{g}\left(-2^{s} t\right)} I_{[0,2 \pi]} \in L_{2}[0,2 \pi]
$$


and the series $\left\{e^{-i t 2^{s} k}, k \in Z\right\}$ is an orthonormal basis for $L_{2}[0,2 \pi]$. An application of the Parseval formula shows that

$$
\sum_{k}\left|\int_{0}^{4 \pi / 3} e^{i t 2^{s} y} \frac{\tilde{\phi}^{(d)}(t)}{\tilde{g}\left(-2^{s} t\right)} e^{-i t 2^{s} k} d t\right|^{2}=\int_{0}^{4 \pi / 3}\left|e^{i t 2^{s} y} \frac{\phi^{(d)}(t)}{\tilde{g}\left(-2^{s} t\right)}\right|^{2} d t=2^{2 s \beta} .
$$

In a similar way, we get that

$$
\sum_{k}\left|\int_{-4 \pi / 3}^{0} e^{i t 2^{s} y} \frac{\tilde{\phi}^{(d)}(t)}{\tilde{g}\left(-2^{s} t\right)} e^{-i t 2^{s} k} d t\right|^{2}=2^{2 s \beta}
$$

Combining the above bounds, it follows that

$$
\sum_{k}\left|\left(H_{s} \phi^{(d)}\right)_{s, k}(y)\right|^{2} \leq 2^{s(2 \beta+1+2 d)}
$$

which in turn implies that

$$
\sum_{k}\left(E\left|Z_{1 k}\right|^{2}\right)^{p / 2} \leq A^{\frac{p}{2}-1} 2^{s(2 \beta+1+2 d)}=2^{s((\beta p+1)+2 d(p-1))} .
$$

Hence,

$$
\begin{aligned}
\sum_{k} E\left|\hat{a}_{s, k}-a_{s, k}\right|^{p} & =\frac{2^{s\left(\frac{1}{2}+\beta+d\right)(p-2)}}{n^{p-1}} \sum_{k} E\left|Z_{1 k}\right|^{2}+\frac{1}{n^{p / 2}} \sum_{k}\left(E\left|Z_{1 k}\right|^{2}\right)^{p / 2} \\
& \leq \frac{2^{s\left(\frac{1}{2}+\beta+d\right)(p-2)} 2^{s(2 \beta+1+2 d)}}{n^{p-1}}+\frac{2^{s(\beta p+1+2 d(p-1))}}{n^{p / 2}} \\
& =\frac{2^{s((\beta p+1)+2 d(p-1))}}{n^{p / 2}}\left(1+\frac{2^{s\left(\frac{p}{2}-1-d(p-2)\right)}}{n^{\frac{p}{2}-1}}\right) .
\end{aligned}
$$

As a consequence of the bound obtained above, it follows that

$$
\begin{aligned}
E\left[\left\|\hat{f}_{X, s}^{(d)}-E\left[\hat{f}_{X, s}^{(d)}\right]\right\|_{p}^{p}\right] & \leq 2^{s\left(\frac{p}{2}-1\right)} \frac{2^{s(\beta p+1)+2 d(p-1)}}{n^{p / 2}}\left(1+\frac{2^{s\left(\frac{p}{2}-1-d(p-2)\right)}}{n^{\frac{p}{2}-1}}\right) \\
& \simeq\left(\frac{2^{s\left(2 \beta+1+4 d \frac{(p-1)}{p}\right)}}{n}\right)^{p / 2} .
\end{aligned}
$$

Choosing $2^{s} \simeq n^{\frac{1-\varepsilon}{1+2 \beta+4 d \frac{(p-1)}{p}}}$ for some $0<\varepsilon<1$, we obtain that

$$
\lim _{n \rightarrow \infty} E\left[\left\|\hat{f}_{X, s}^{(d)}-E\left[\hat{f}_{X, s}^{(d)}\right]\right\|_{p}^{p}\right]=0
$$

Combining the relations (4.8) and (4.10), we obtain that

$$
\lim _{n \rightarrow \infty} E\left[\left\|\hat{f}_{X, s}^{(d)}-f_{X}^{(d)}\right\|_{p}^{p}\right]=0
$$


by the inequality

$$
\|U+V\|_{p} \leq\|U\|_{p}+\|V\|_{p}
$$

for $U, V \in L_{p}(R)$. This proves the $L_{p}$-consistency of the estimator $\hat{f}_{X, s}^{(d)}$ for estimating the derivative $f_{X}^{(d)}$.

Remarks. We have discussed the problem of estimation of derivative of a density function in the presence of independent additive noise whose distribution is known using linear wavelet estimators. It is not clear how to estimate the original density or its derivative if the error density is also unknown. Another problem is to study adaptive nonlinear wavelet estimators of derivative of a density in the presence of known or unknown independent additive noise and to construct shape preserving estimators. These problems need investigation.

\section{Acknowledgment}

This work was supported under the scheme "Ramanujan Chair Professor" at the CR Rao Advanced Institute for Mathematics, Statistics and Computer Science, Hyderabad, India.

\section{References}

Andersen, K. and Hansen, M. (2001). Multiplicative censoring: Density estimation by a series expansion. Journal of Statistical Planning and Inference 98, 137-155. MR1860231

Antoniadis, A., Gregoire, G. and McKeague, I. W. (1994). Wavelet methods for curve estimation. Journal of the American Statistical Association 89, 1340-1353. MR1310225

Asgharian, M., Carone, M. and Fakoor, V. (2012). Large-sample study of the kernel density estimation under multiplicative censoring. The Annals of Statistics 40, 159-187. MR3013183

Chaubey, Y. P., Chesneau, C. and Doosti, H. (2011). On linear wavelet density estimation: Some recent developments. Journal of the Indian Society of Agricultural Statistics 65, 169-179. MR2868127

Chaubey, Y. P., Chesneau, C. and Doosti, H. (2015). Adaptive wavelet estimation of a density from mixtures under multiplicative censoring. Statistics 49, 638-659. MR3349084

Chaubey, Y. P., Doosti, H. and Prakasa Rao, B. L. S. (2006). Wavelet based estimation of the derivatives of a density with associated variables. International Journal of Pure and Applied Mathematics 27, 97-106. MR2214707

Chaubey, Y. P., Doosti, H. and Prakasa Rao, B. L. S. (2008). Wavelet based estimation of the derivatives of a density for a negatively associated process. Journal of Statistical Theory and Practice 2, 453-463. MR2528792

Chesneau, C. (2013). Wavelet estimation of a density in a GARCH-type model. Communications in Statistics Theory and Methods 42, 98-117. MR3004648

Chesneau, C. and Doosti, H. (2012). Wavelet linear density estimation for a GARCH model under various dependence structures. Journal of the Iranian Statistical Society 11, 1-21. MR2899367

Daubechies, I. (1988). Orthogonal bases of compactly supported wavelets. Communications on Pure and Applied Mathematics 41, 909-996. MR0951745 
Daubechies, I. (1992). Ten Lectures on Wavelets. Philadelphia: SIAM. MR1162107

Donoho, D., Johnstone, I., Kerkyacharian, G. and Picard, D. (1996). Density estimation by wavelet thresholding. The Annals of Statistics 24, 508-539. MR1394974

Fan, J. and Koo, J. (2002). Wavelet deconvolution. IEEE Transactions on Information Theory 48 , 734-747. MR1889978

Geng, Z. and Wang, J. (2015). The mean consistency of wavelet density estimators. Journal of Inequalities and Applications 2015, Article ID 111. MR3326219

Hardle, W., Kerkycharian, G., Picard, D. and Tsybakov, A. (1998). Wavelets, Approximations, and Statistical Applications. Lecture Notes in Statistics 129. New York: Springer. MR1618204

Hosseinioun, N. (2016). Wavelet-based density estimation in presence of additive noise under various dependence structures. Advances in Pure Mathematics 6, 7-15.

Leblanc, F. (1996). Wavelet linear density estimator for a discrete-time stochastic process: $L_{p}$-losses. Statistics \& Probability Letters 27, 71-84. MR1394179

Li, R. and Liu, Y. (2014). Wavelet estimations for a density with some additive noises. Applied and Computational Harmonic Analysis 36, 416-433. MR3175086

Li, Q. and Racine, J. S. (2007). Nonparametric Econometrics: Theory and Practice. Princeton: Princeton University Press. MR2283034

Lounici, K. and Nickl, R. (2011). Global uniform risk bounds for wavelet deconvolution estimators. The Annals of Statistics 39, 201-231. MR2797844

Mallat, S. G. (1989). A theory for multiresolution signal decomposition: The wavelet representation. IEEE Transactions on Pattern Analysis and Machine Intelligence 11, 674-693.

Prakasa Rao, B. L. S. (1983). Nonparametric Functional Estimation. Orlando: Academic Press. MR0740865

Prakasa Rao, B. L. S. (1996). Nonparametric estimation of the derivatives of a density by the method of wavelets. Bulletin of Informatics and Cybernetics 28, 91-100. MR1399182

Prakasa Rao, B. L. S. (1999a). Nonparametric functional estimation: An overview. In Asymptotics: Nonparametrics and Time Series (S. Ghosh, ed.) 461-509. New York: Marcel Dekker Inc. MR1724706

Prakasa Rao, B. L. S. (1999b). Estimation of the integrated squared density derivative by wavelets. Bulletin of Informatics and Cybernetics 31, 47-65. MR1700556

Prakasa Rao, B. L. S. (2000). Nonparametric estimation of partial derivatives of a multivariate probability density by the method of wavelets. In Asymptotics in Statistics and Probability: Festschrift for George G. Roussas (M. L. Puri, ed.) 321-330. Amsterdam: VSP. MR1945441

Prakasa Rao, B. L. S. (2003). Wavelet linear density estimation for associated sequences. Journal of the Indian Statistical Association 41, 369-379. MR2102002

Prakasa Rao, B. L. S. (2010). Wavelet linear estimation for derivatives of a density from observations of mixtures with varying mixing proportions. Indian Journal of Pure and Applied Mathematics 41, 275-291. MR2650112

Prakasa Rao, B. L. S. (2017). Wavelet estimation for derivative of a density in a GARCH-type model. Communications in Statistics Theory and Methods 46, 2396-2410. MR3576723

Rosenthal, H. P. (1970). On the subspaces of $L^{p},(p>2)$ spanned by sequences of independent random variables. Israel Journal of Mathematics 8, 273-303. MR0271721

Shirazi, E., Chaubey, Y. P., Doosti, H. and Nirumand, H. A. (2012). Wavelet based estimation for the derivative of a density by block thresholding under random censorship. Journal of the Korean Statistical Society 41, 199-211. MR3255291

Strang, G. (1989). Wavelets and dilation equations: A brief introduction. SIAM Review 31, 614-627. MR1025484

Tribouley, K. (1995). Practical estimation of multivariate densities using wavelet methods. Statistica Neerlandica 49, 41-62. MR1333178

Vardi, Y. (1989). Multiplicative censoring, renewal processes, deconvolution and decreasing density: Nonparmetric estimation. Biometrika 76, 751-761. MR1041420 
Vardi, Y. and Zhang, C. H. (1992). Large sample study of empirical distributions in a random multiplicative censoring model. The Annals of Statistics 20, 1022-1039. MR1165604

Vidakovic, B. (1999). Statistical Modeling by Wavelets. New York: Wiley. MR1681904

Walter, G. and Ghorai, J. (1992). Advantages and disadvantages of density estimation with wavelets. In Graphics and Visualization: Proceedings of the 24th Symposium on the Interface (H. J. Newton, ed.). Computing Science and Statistics 24, 234-243. Fairfax Station: Interface Foundation of North America.

CR Rao Advanced Institute of Mathematics, Statistics and Computer Science Hyderabad

India

E-mail: blsprao@gmail.com 